\title{
BMJ Open Persistence of patient and family experiences of critical illness
}

\author{
Eliotte L Hirshberg (10 , 1,2,3,4 Jorie Butler, ${ }^{1,5,6}$ Morgan Francis, ${ }^{2}$ Francis A Davis, ${ }^{7}$ \\ Doriena Lee, ${ }^{7,8}$ Fahina Tavake-Pasi, ${ }^{7}$ Edwin Napia, ${ }^{7}$ Jeanette Villalta, ${ }^{7}$ \\ Valentine Mukundente, ${ }^{7}$ Heather Coulter, ${ }^{7,8}$ Louisa Stark, ${ }^{7,8}$ Sarah J Beesley, ${ }^{1,2}$ \\ James F Orme, ${ }^{1,2,3}$ Samuel M Brown, ${ }^{1,2,3}$ Ramona O Hopkins ${ }^{1,2,9}$
}

To cite: Hirshberg EL, Butler J, Francis $\mathrm{M}$, et al. Persistence of patient and family experiences of critical illness. BMJ Open 2020;10:e035213. doi:10.1136/ bmjopen-2019-035213

- Prepublication history and additional material for this paper are available online. To view these files, please visit the journal online (http://dx.doi. org/10.1136/bmjopen-2019035213).

ELH and JB are joint first authors.

Received 23 0ctober 2019 Revised 10 March 2020 Accepted 12 March 2020
Check for updates

(c) Author(s) (or their employer(s)) 2020. Re-use permitted under CC BY-NC. No commercial re-use. See rights and permissions. Published by BMJ.

For numbered affiliations see end of article.

Correspondence to Dr Eliotte L Hirshberg; ellie.hirshberg@imail.org

\section{ABSTRACT}

Objective To investigate: (1) patient and family experiences with healthcare and the intensive care unit (ICU); (2) experiences during their critical illness; (3) communication and decision making during critical illness; (4) feelings about the ICU experience; (5) impact of the critical illness on their lives; and (6) concerns about their future after the ICU.

Design Four semistructured focus group interviews with former ICU patients and family members.

Settings Multicultural community group and local hospitals containing medical/surgical ICUs.

Participants Patients and family who experienced a critical illness within the previous 10 years.

Interventions None.

Measurements and main results Four separate focus groups each lasting a maximum of 150 min and consisting of a total of 21 participants were held. Focus groups were conducted using a semistructured script including six topics relating to the experience of critical illness that facilitated deduction and the sorting of data by thematic analysis into five predominant themes. The five main themes that emerged from the data were: (1) personalised stories of the critical illness; (2) communication and shared decision making, (3) adjustment to life after critical illness, (4) trust towards clinical team and relevance of cultural beliefs and (5) end-of-life decision making. Across themes, we observed a misalignment between the medical system and patient and family values and priorities.

Conclusions The experience of critical illness of a diverse group of patients and families can remain vivid for years after ICU discharge. The identified themes reflect the strength of memory of such pivotal experiences and the importance of a narrative around those experiences. Clinicians need to be aware of the lasting effects of critical illness has on patients and families.

\section{INTRODUCTION}

Critical illness and treatment in an intensive care unit (ICU) is an experience that is fraught with uncertainty and stress for patients and family. ${ }^{1-3}$ It also can be a disruptive and emotional event. ${ }^{4-6}$ During times of stress and fear, clear communication is important..$^{5}$ Previous experiences and cultural norms may influence perceptions, communication, behaviour and medical choices that then

\section{Strengths and limitations of this study}

This study is one of a few qualitative studies to assess patient and family perceptions of their intensive care unit (ICU) experience.

- We performed a descriptive qualitative focus group study of patient and family perceptions analysed using thematic analysis.

- The diverse and multicultural participant group enhances the generalisability of the results.

- The small number of participants may limit the generalisability of the findings.

- Some participants were interviewed up to 10 years after their ICU experience, which may have introduced recall bias.

shape a critical illness experience. ${ }^{8}$ Family satisfaction with communication and shared decision making is influenced by trust in providers and is directly related to the feelings patients and family members have after critical illness. $^{29}$

Several factors including the quality of clinicians' communication and shared decision making with patients and family can influence their experiences of critical illness. ${ }^{5-7} 10$ Contextual factors such as cultural background, educational level and religious beliefs often impact communication and interpersonal interactions. Even a simple medical communication may be perceived in diverse ways. ${ }^{611}$ An understanding of patient and family experiences, shaped by cultural beliefs, medical knowledge and communication with clinicians is a critical step in efforts to improve patient and family ICU satisfaction. ${ }^{13}$

Narrative descriptions of lived encounters are powerful reflections of how individuals process an experience. ${ }^{14} 15$ Simply describing an experience may trigger acute and longterm stress. ${ }^{16}{ }^{17}$ Similarly, critical illness may be a pivotal experience that shapes stress and emotional well-being. To advance 
patient-centred care, it is crucial to understand patient and family experiences of critical illness.

In this qualitative study, we conducted focus groups with an ethnically diverse sample of patients and family with a history of critical illness and admission to the ICU. Our objective was to understand their experiences, communication preferences, decision making and feelings during the ICU and explore how the critical illness impacted patients and family and their concerns about navigating their future after critical illness.

\section{METHODS}

\section{Participants}

We recruited participants who met the following study inclusion criteria: age 18 years or older who had been hospitalised in the ICU or had a family member hospitalised in the ICU within the preceding 10 years. Participants were contacted via telephone by a research assistant (MF) and selected using purposive sampling ${ }^{18}$ of eligible candidates from Intermountain Medical Center and Community Faces of Utah (CFU). At Intermountain Medical Center, clinicians and researchers identified patients or family members of patients who had a previous admission to the ICU. At CFU, we recruited participants who were admitted or had a family member admitted to an ICU in the last 10 years. CFU is a partnership among the University of Utah, the Utah Department of Health and leaders of five ethnically diverse communities in the state of Utah (African refugee/immigrant, African-American, American Indian/Alaskan Native, Hispanic/Latino and Pacific Islander); CFU is dedicated to improving health equity and access with local healthcare partners. ${ }^{19}$ The leaders of the five communities queried their members to identify individuals or family who had a critical illness or had been admitted to an ICU. Participants who met study inclusion criteria were provided with oral and written information about the study. Written informed consent was obtained prior to participation in the focus groups.

\section{Patient and public involvement}

The study objectives were developed by the research team and refined by the ICU Patient and Family Advisory Counsel at Intermountain Medical Center. Patients were not directly involved in the recruitment to or conduct of the study. Members of CFU participated in data coding and member checking and are coauthors or acknowledged where appropriate.

\section{Data collection}

All participants: were asked about: (1) patient and family experiences with healthcare and the ICU (eg, sudden illnesses vs chronic conditions), (2) their experience during the ICU stay, (3) communication and decision making around critical illness, (4) feelings about the ICU experience, (5) the impact of critical illness on their lives and (6) concerns about their future after the ICU. The focus group script was developed through an iterative process, reviewed and agreed on by the research team prior to the first focus group.

\section{Researchers and participant relationships}

An experienced qualitative researcher and health psychologist PhD (JB) employed at the University of Utah and the Veterans Affairs Hospital and a board-certified critical care physician employed at Intermountain Healthcare (ELH) facilitated the discussions. Neither researcher had a pre-existing relationship with any of the volunteer participants. The introduction portion of each focus group included an introduction of each researcher and a description of their reasons for conducting the interviews. Each researcher disclosed their interest in learning about the patient and family experience in the ICU. Minimal characteristics about each facilitator were revealed to minimise exposure bias.

\section{Setting and script}

Focus groups were conducted using a semistructured interview script (see online supplementary appendix A) between March 2017 and May of 2017. The focus group sessions were conducted in either conference rooms at the study hospital or for CFU participants, in a conference room at their meeting facility in Salt Lake City, Utah. In addition to the focus group participants and interviewers, research staff from CFU and/or Intermountain Medical Center observed the focus group discussions. The interviews continued until no new information relevant to the research questions emerged from the participants. Focus group sessions were audio recorded and professionally transcribed; there were approximately 10 hours of material from all the focus groups. Transcriptions included only deidentified information.

\section{Qualitative analysis}

After the completion of all focus groups, transcripts were loaded into NVivo 8.0 for content analysis and coding. Data were analysed using thematic analysis. ${ }^{20}$ The data analysis team included a practising critical care physician, neuroscientist, health psychologist and CFU members. Each member of the analytic team (ROH, JB and ELH) individually reviewed the focus group transcript and independently coded all transcripts. A series of codes were identified, tightly linked to the participant quotes. The researchers then met together and resolved coding discrepancies through a consensus approach and determined final codes. Each of the final codes that emerged from the data were then grouped together into cohesive themes. Finally, central themes that repeatedly emerged from the data were identified. Selected portion of the deidentified transcripts with assigned codes and themes were member checked by both by CFU researchers and two participants of the focus groups for comment and theme clarity.

\section{RESULTS}

Twenty-one adults from diverse cultural backgrounds participated in four separate heterogenous focus groups, 
each lasting a maximum of $150 \mathrm{~min}$. We identified five major themes. A description of each theme and representative sample quotes are provided in table 1.

\section{Theme 1: personalised ICU stories}

Theme 1 consisted of codes based on quotes that related to the patient and family narratives of their critical illness experience. The narratives included information about perceptions of the medical information they received and their interactions with clinical staff, and their perceived role during the ICU encounter. Self-described roles during the ICU from the participants included: caregiver, observer, advocate, decision maker and sometimes leader of interactions with the clinical team. The stories persisted for months to years and provided context within which to interpret participants' experience and seem to help them cope with the emotional distress associated with the ICU encounter.

In one example, the participant story notes a need to compartmentalise coping:

... I had to compartmentalize it and just say, okay, we're going to get through the next threehours and then we are going to see what happens... I just have this switch in my brain that I flip into hospital mode... I try not to think too far ahead, just deal with the right now, and we will deal with what comes when it gets here...

In another example, a different participant (a patient) describes their critical illness as being a job:

So, really it was like a job trying to get well, watching the television and listening to what the doctors and it was so unbelievable what I learned during the time I was flat on my back for those 3 weeks with the determination to come out, not knowing that I would never have that job anymore.

\section{Theme 2: communication and shared decision making}

Participants reported both positive and negative communications with care teams in the ICU. Positive communication occurred when they were involved in making significant decisions with input from the clinical team, when they had access to complete information and when patients' wishes were honoured. In contrast, other participants reported negative experiences when communication was vague or unclear, or when patient priorities seemed to be ignored by clinical staff. This suggests misalignment between institution and participant priorities.

In one experience a participant describes a perceived insensitive communication:

I just thought, he had a stomach ache.... and when we arrived the doctors asked me how much we wanted them to do to save his life... I want you to do everything you can. So, I think that for me that was just one question that always lingered in my mind that somebody would actually say, you know, how much do you want us to do for your family member because it just seemed like that was a dumb question...

In this case, the standard question of asking do not resuscitate status was perceived by family as an inappropriate question.

There were indications that facets of an academic health system were difficult for participants:

[After visits from several trainees] ...finally, the one came in and I said, if one more of you come in here and ask me what brought us here today, I am going to punch you. I'd better have a doctor in front of me in 15 min or I will literally punch the next resident who walks in the door.

The distress noted above at numerous providers in an academic medical centre appeared to be at odds with patient and family desires about communication. In an example of unclear communication and a potential misunderstanding, the family believed that the patient was kept alive for 'study':

... [M] y sister was, I think she was dead ... they had her up at the [specific hospital] on life support and one day they would say oh, she's doing okay, and then the next day they would say, it doesn't look so good, and this went on for a couple of weeks and finally I confronted the head of the neurology department ... you need to tell us what is going on and you had better not be keeping her alive just to study her case.... the director stopped and said, you had better call the family together, so they were keeping her alive to study her case because the director is the only one that had ever seen a case like that and so, none of the other neurologists had seen that, and so that is why they... I mean, I can see them wanting to study it, but I couldn't see them doing to my family what they were doing, so. So that wasn't such a good experience...

Often participants' perceptions and descriptions (like the one noted above) of clinical situations did not reflect established clinical practice related to disease processes, suggesting that patients' and family's recollection of events during critical illness differed from the intended communication of the medical staff.

\section{Theme 3: adjustment to life after critical illness (the 'new normal')}

Patients and family reported intense feelings about their experiences even months and years after the critical illness. These feelings included helplessness, loss of control, uncertainty and gratitude. The feeling of being alone, even 'invisible', was common. Strong feelings around the transition out of the ICU—whether by death or clinical improvement-were common, highlighting the grief that occurs when a life is changed and altering future dreams and expectations. Participants described their life postcritical illness as the 'new normal'. The new normal included new financial burdens including the 

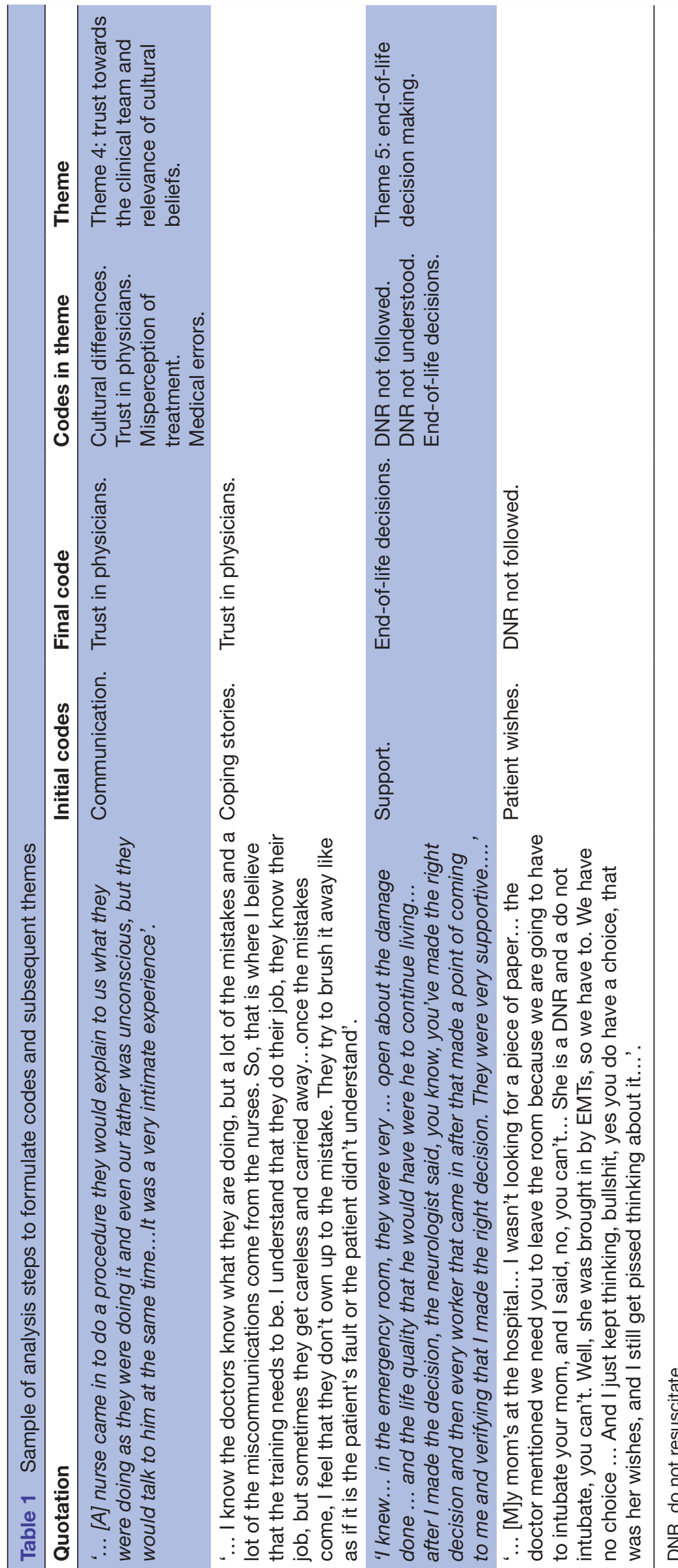
inability to get life insurance, need to return to work or the burden of medical bills.

In one example, a participant felt very alone in the waiting room:

So... that was hard, because it wasn't just him, I had a lot to process too and I just.... It's not like I can call up a bunch of people and say hey, guess what the latest thing on my plate is, because, it was a situation where his privacy had to be respected, so I couldn't talk about it, and it was just feeling invisible just really compounded that feeling of just helplessness and being alone.

Two other participants reported concerns around financial struggles or described the many ways life changed after the critical illness:

... Some stress around bills, some stress around what's going to happen with the recovery, some stress around not knowing what to expect...

... He is still in cognitive therapy and physical therapy and his personality did change, which wasn't bad, but it was just different and so that has been an adjustment and I think the long term of it, I didn't think we'd be here still...., this is our new normal and so we're just trying to now recreate a new dream so to speak...

\section{Theme 4: trust towards the clinical team and relevance of cultural beliefs}

Participants' expectations and sense of trust influenced their perceptions of partnership with the clinical staff. When medical errors were perceived to have occurred, pervasive mistrust resulted. Cultural background also influenced experiences and participant ideas of clinical team roles (doctors or nurses) that could cause frustration when the experiences were different than the expectations of the patient or family. Participants openly shared their accolades, frustrations and their clinician role ideas. In one case in which trust was built, the family member participant said:

... [A] nurse came in to do a procedure they would explain to us what they were doing as they were doing it and even our father was unconscious, but they would talk to him at the same time... It was a very intimate experience.

In contrast another family member participant said:

... I know the doctors know what they are doing, but a lot of the mistakes and a lot of the miscommunications come from the nurses. So, that is where I believe that the training needs to be. I understand that they do their job, they know their job, but sometimes they get careless and carried away... once the mistakes come, I feel that they don't own up to the mistake. They try to brush it away like as if it is the patient's fault or the patient didn't understand.
In addition, cultural experiences influenced expectations:

[S]o I am stressed out because everyone is talking about HIPAA won't allow me to tell you if the patient is here and that HIPAA won't allow me to tell you this... but in the African American community the (person in my community leader role) is the person that everybody turns to and expects to know what is going on. So it is very stressful when you can't get the information that you need. In this recent case, I was the person that the family said is their spokesperson, is their person to get the information that you need.

\section{Theme 5: end-of-life decision making}

End-of-life stories were generally positive, and stories about anticipated deaths were more favourable than deaths that occurred suddenly. Favourable stories relied on self-identified understanding of the medical diagnosis and what seemed to be an affirmation of the decision from the clinicians. In contrast, negative experiences were characterised by the perception that patient wishes were not fulfilled.

A clear understanding of patient preferences gave participants confidence that they knew what should happen.

I knew... in the emergency room, they were very ... open about the damage done ... and the life quality that he would have were he to continue living... after I made the decision, the neurologist said, you know, you've made the right decision and then every worker that came in after that made a point of coming to me and verifying that I made the right decision. They were very supportive....

In contrast, there was frustration when patient preferences were not adhered to as another participant stated:

... [M]y mom's at the hospital... I wasn't looking for a piece of paper... the doctor mentioned we need you to leave the room because we are going to have to intubate your mom, and I said, no, you can't... She is a DNR and a do not intubate, you can't. Well, she was brought in by EMTs, so we have to. We have no choice ... And I just kept thinking, bullshit, yes you do have a choice, that was her wishes, and I still get pissed thinking about it...

The dissatisfaction with the end-of-life experience continued to be an ongoing issue for this participant.

\section{DISCUSSION}

In this study, we explored the experiences of a diverse group of patients and family members in the USA following a critical illness. The five themes we identified reflect the persistence of memory of such pivotal experiences that happened up to 10 years previously. The stories also reflect the construction of a narrative around these 
experiences. The narrative may reflect personal characteristics of the participants ${ }^{14}$ and the sense-making process ${ }^{21}$ they have engaged in related to their experience. For example, personal characteristics such as a conception of the self as efficacious and powerful can influence the narrative description of the personal agency experienced while advocating on behalf of a patient.

The experience of critical illness was uniformly reported as stressful for our participants even if survival was the ultimate outcome. Our data reinforce the importance of recognising ${ }^{22}$ and addressing postintensive care syndrome (PICS) in survivors of critical illness and their family members after an ICU admission and suggest the sequelae persists for years. Stories were frequently characterised by stressful events around communication including shared decision-making encounters. Family member participants sometimes felt they were the only advocate for the patient within an indifferent medical system resulting in added stress.

Our findings suggest that many patients and families need help and support beyond traditional medical follow-up after an ICU admission, which is supported in work by others. ${ }^{23}$ We found that participants may have difficulty adjusting to a 'new normal' and possibly lack resilience. Their ICU experience appears to shape the feelings they have about both the critical illness and their transition back to a 'normal life', consistent with other narrative studies in ICU populations. ${ }^{24-26}$ Post-ICU populations sometimes describe a transformation in the years following an ICU admission. ${ }^{26}$ This type of transformation is consistent with positive growth and may be linked to building resilience and is similar to the descriptions of emotional resilience described in other survivors of traumatic experiences. ${ }^{27} 28$ Many individuals reported that they found new ways to thrive within their "new normal', suggesting the importance of further investigation of resilience training and interventions to facilitate post-traumatic growth in ICU survivors and their family. Acknowledgement by the medical community of the potential challenges after and ICU admission and recommendations to use experience diaries may help reduce depression and anxiety and improve quality of life. ${ }^{25} 29$

Critical to our findings were the range of communication and decision-making scenarios that characterised the critical illness experience across themes. Family surrogate decision makers weigh multiple factors beyond the wishes of the patients while engaging in the shared decision process. ${ }^{40}$ It follows that surrogate decision makers might look for validation of their recommendations from clinicians when discussing important treatment decisions. This would make misalignment of priorities even more impactful. The persistent need for surrogate decision makers to feel validated in their self-appraisal of ICU events by clinicians is consistent with the commonly accepted stress appraisal conditions in other psychology literature. ${ }^{31}$

In our data, we frequently saw statements that seemed to reflect misunderstandings of clinical course or treatment decisions. The recollections of providers' expected roles and presumed intent reflected frustration with the care processes and workflow at academic medical centres. Both misunderstandings and frustrations likely contributed to increased stress and confusion. ${ }^{71224}$ These misperceptions may relate to health literacy that has been found to predict successful communication and navigation of health services. ${ }^{32} 33$ Another potential explanation of misperceptions is the influence of past experiences, some of which may be impacted by culture. To our knowledge, the misalignment between clinical processes and patient experience is a novel finding. Participants seemed to perceive that the medical system is operating under its own agenda (eg, trainees providing medical care; introducing 'code status' conversations prematurely), whereas the patient or family member desired a more collaborative relationship that focused on their specific situation.

A misalignment highlights the need for clinicians to better understand family expectations both about the anticipated medical course and about the nature of teamwork with clinicians. Medical providers need to be aware that these misunderstandings may be common and have long-lasting impact on patients and family recovering from an ICU admission.

A greater understanding of patient and family past experiences and how that might shape expectations can create a framework for communication going forward, which is critical to partnerships among patients and family and the medical system. ${ }^{34}$

We found that culture and trust influence effective medical partnerships and decision making, thereby shaping experiences. ${ }^{35}$ In our study and work by others', cultural influences appeared to span beyond simple failures of communication, as people of different cultural groups often had different community structures and expectations. ${ }^{36}$ This was supported in our results in one case where a key community figure was not allowed to participate in information sharing due to Health Insurance Portability and Accountability Act restrictions. Similarly, there are indications that conversations about 'code status' may be perceived very differently based on the cultural background of the patient and family member. ${ }^{37}$ Our study supports the observation from others that during times of stress and fear, trauma-informed communication, cultural understanding and validation are crucial. $^{5} 7$ Perhaps, medical training should evolve to encompass teaching the communication training and techniques such as Connect, Listen, Empathise, Align, Respect conversations developed at Henry Ford Health System or VitalTalk (http:/ /www.VitalTalk.org) ${ }^{38}{ }^{39}$ Both programmes focus on communication that elicits patient and family values.

The ICU experiences at the end of life largely reflect survivors' participation in and understanding about their loved ones wishes. End-of-life stories were mostly positive among our participants and reiterated the importance of participant self-identified understanding of the medical diagnosis with affirmation from clinicians. 
Previous studies also report the importance of communication and family understanding in end-of-life decisions. ${ }^{6} 384041$ Our data support the idea that expressions of non-abandonment and empathy are strategies that increase family satisfaction ${ }^{12} 41$ and underscores the power of healthcare provider acceptance and affirmation of patient and family ideas. Therefore, there is an imperative to shift current communication during critical illness to a more tailored and personalised approach that educates clinicians about connection and cultural expectations for all communications including end of life.

A realignment of institutional and medical community priorities with a more individualised approach to care is a challenge across the USA. We suggest that this realignment begins with medical training and must extend to all care providers and hospital administrators. This will require a shift in paradigm where 'personalised care' is focused around patient priorities in conjunction with or instead of genetic predisposition. ${ }^{42}$ The inherent opportunity presented by this realignment within the ICU could be a profound alteration of stressful negative experiences and the morbidity associated with PICS. Patients and families may benefit from clinical tools that facilitate clearer communication on the part of clinicians with a focus on patient priorities. Such tools should be trauma informed and focus on health system literacy and can be built to support joint responsibility between clinicians and patients or family members and can ensure more complete understanding of the critical illness experience. These shared decision-making tools could be developed to support ICU decisions such as those that are operating in other domains and considered for development in the ICU. ${ }^{43-45}$ In addition, patient and family educational information could be structured to reduce stress burden from misunderstandings. All will likely enhance patient and family satisfaction.

The limitations of this study include those common to qualitative research including small sample size and that some perspectives may have been missed. Participants were recruited from a purposive sample, and we did not track those who might have had opportunity but chose not to participate. Some participants were interviewed years after their ICU admission, which may have contributed to recall bias for poor or positive experiences. Though the vivid recollection of these events may also be considered a strength. Lastly, qualitative methods cannot distinguish the impact of the ICU admission from the psychological impact of the illness itself.

This study is one of a few qualitative studies to assess patient and family perceptions of their ICU experience. A second strength of our study is its inclusion of multicultural participants. In contrast to most prior qualitative studies of ICU experience in the USA, we recruited participants from a diverse array of ethnic communities and their community leaders to increase the generalisability of our findings. Another strength of our study is our inclusion of participants several years after the ICU experience, which helps us gain a better sense for the persistence of the experience of critical illness even years after the ICU admission. ${ }^{31}$ Distinguishing the impact of the critical illness and ICU admission from the psychological impact of the illness itself are both aspects that likely merit attention in the development of optimal approaches to support patients, families and clinicians in the ICU.

\section{CONCLUSIONS}

Qualitative data suggest that the experiences of an ethnically and culturally diverse group of patients and family members during a critical illness remain vivid years later. The five themes identified reflect strong and persistent memories of such pivotal experiences and the construction of a narrative around the experiences. The experience of critical illness was uniformly reported as stressful for our participants, and there is a perception of misalignment of institutional and patient/family priorities. Future work could examine the effectiveness of shared communication tools to support engaged decision making.

\section{Author affiliations}

${ }^{1}$ Center for Humanizing Critical Care, Internountain Medical Center, Murray, Utah, USA

${ }^{2}$ Department of Medicine, Division of Pulmonary and Critical Care, Intermountain Healthcare, Murray, Utah, USA

${ }^{3}$ Department of Medicine, University of Utah School of Medicine, Salt Lake City, Utah, USA

${ }^{4}$ Pediatrics, University of Utah, School of Medicine, Salt Lake City, Utah, USA ${ }^{5}$ Department of Geriatrics, Univeristy of Utah, Salt Lake City, Utah, USA

${ }^{6}$ Geriatrics Research Education and Clinical Center, Veterans Affairs Medical Center (VAMC, Salt Lake City, Utah, USA

${ }^{7}$ Community Faces of Utah, Salt Lake City, USA

${ }^{8}$ University of Utah Health Sciences Center, Salt Lake City, Utah, USA

${ }^{9}$ Department of Psychology and Neuroscience, Brigham Young University, Provo, Utah, USA

\section{Twitter Eliotte L Hirshberg @elhrishberg}

Contributors Conception (ELH, JB and ROH), data acquisition (ELH, JB, MF, JFO, EN, FAD, DL, FT-P, EN, JV, VM, HC and LS), data analysis (ELH, JB, ROH, EN and FAD), writing manuscript (ELH, JB, ROH, SMB and SJB), revising manuscript for important intellectual content (all authors) and approval of final copy (all authors)

Funding This study was funded by departmental funds/Intermountain Research foundation.

Disclaimer The views expressed in this article are those of the authors and do not necessarily reflect the position or policy of the Department of Veterans Affairs or the US government.

\section{Competing interests None declared.}

Patient and public involvement Patients and/or the public were involved in the design, or conduct, or reporting, or dissemination plans of this research. Refer to the Methods section for further details.

Patient consent for publication Not required.

Ethics approval All study procedures were approved by the Intermountain Healthcare Institutional Review Board (\#1024730). Requests for deidentified data from qualified researchers with appropriate ethics board approvals and relevant data use agreements will be processed by the Intermountain Office of Research, officeofresearch@imail.org.

Provenance and peer review Not commissioned; externally peer reviewed.

Data availability statement Data are available on reasonable request. Dataset and coding dictionary can be made available on request from the corresponding author. 
Open access This is an open access article distributed in accordance with the Creative Commons Attribution Non Commercial (CC BY-NC 4.0) license, which permits others to distribute, remix, adapt, build upon this work non-commercially, and license their derivative works on different terms, provided the original work is properly cited, appropriate credit is given, any changes made indicated, and the use is non-commercial. See: http://creativecommons.org/licenses/by-nc/4.0/.

Author note Both the SRQR and the CODEQ checklists were completed for methodological reporting accuracy.

ORCID iD

Eliotte L Hirshberg http://orcid.org/0000-0002-3838-2679

\section{REFERENCES}

1 Davydow DS, Zatzick D, Hough CL, et al. A longitudinal investigation of alcohol use over the course of the year following medical-surgical intensive care unit admission. Psychosomatics 2013;54:307-16.

2 Epstein EG, Wolfe K. A preliminary evaluation of trust and shared decision making among intensive care patients' family members. Appl Nurs Res 2016;32:286-8.

3 Petrinec AB, Mazanec PM, Burant CJ, et al. Coping strategies and posttraumatic stress symptoms in Post-ICU family decision makers. Crit Care Med 2015;43:1205-12.

4 Li L, Nelson JE, Hanson LC, et al. How surrogate decision-makers for patients with chronic critical illness perceive and carry out their role. Crit Care Med 2018;46:699-704.

5 Heyland DK, Rocker GM, O'Callaghan CJ, et al. Dying in the ICU: perspectives of family members. Chest 2003;124:392-7.

6 Brooks LA, Manias E, Nicholson P. Communication and decisionmaking about end-of-life care in the intensive care unit. Am J Crit Care 2017;26:336-41.

7 Heyland DK, Cook DJ, Rocker GM, et al. Decision-making in the ICU: perspectives of the substitute decision-maker. Intensive Care Med 2003;29:75-82.

8 Davydow DS, Zatzick D, Hough CL, et al. A longitudinal investigation of posttraumatic stress and depressive symptoms over the course of the year following medical-surgical intensive care unit admission. Gen Hosp Psychiatry 2013;35:226-32.

9 Fisher M, Ridley S. Uncertainty in end-of-life care and shared decision making. Crit Care Resusc 2012;14:81-7.

10 Kon AA, Davidson JE, Morrison W, et al. Shared decision making in ICUs: an American College of critical care medicine and American thoracic Society policy statement. Crit Care Med 2016;44:188-201.

11 Elliott AM, Alexander SC, Mescher CA, et al. Differences in physicians' verbal and nonverbal communication with black and white patients at the end of life. J Pain Symptom Manage 2016;51:1-8.

12 Wilson ME, Akhoundi A, Krupa AK, et al. Development, validation, and results of a survey to measure understanding of cardiopulmonary resuscitation choices among ICU patients and their surrogate decision makers. BMC Anesthesiol 2014;14:15.

13 Tramm R, llic D, Murphy K, et al. Experience and needs of family members of patients treated with extracorporeal membrane oxygenation. J Clin Nurs 2017;26:1657-68.

14 McAdams DP, McLean KC. Narrative identity. Curr Dir Psychol Sci 2013;22:233-8.

15 Adler JM, Skalina LM, McAdams DP. The narrative reconstruction of psychotherapy and psychological health. Psychother Res 2008;18:719-34.

16 Pennebaker JW. Putting stress into words: health, linguistic, and therapeutic implications. Behav Res Ther 1993;31:539-48.

17 Berry DS, Pennebaker JW. Nonverbal and verbal emotional expression and health. Psychother Psychosom 1993;59:11-19.

18 Benoot C, Hannes K, Bilsen J. The use of purposeful sampling in a qualitative evidence synthesis: a worked example on sexual adjustment to a cancer trajectory. BMC Med Res Methodol 2016;16:21.

19 Simonsen SE, Digre KB, Ralls B, et al. A gender-based approach to developing a healthy lifestyle and healthy weight intervention for diverse Utah women. Eval Program Plann 2015;51:8-16.

20 Braun V, Clarke V. Using thematic analysis in psychology. Qual Res Psychol 2006;3:77-101.

21 Adler JM, Turner AF, Brookshier KM, et al. Variation in narrative identity is associated with trajectories of mental health over several years. J Pers Soc Psychol 2015;108:476-96.
22 Vlake JH, van Genderen ME, Schut A, et al. Patients suffering from psychological impairments following critical illness are in need of information. J Intensive Care 2020;8:6.

23 Haines KJ, Sevin CM, Hibbert E, et al. Key mechanisms by which post-ICU activities can improve in-ICU care: results of the International thrive collaboratives. Intensive Care Med 2019:45:939-47.

24 White DB, Braddock CH, Bereknyei S, et al. Toward shared decision making at the end of life in intensive care units: opportunities for improvement. Arch Intern Med 2007;167:461-7.

25 Flynn K, Daiches A, Malpus Z, et al. 'A post-transplant person': Narratives of heart or lung transplantation and intensive care unit delirium. Health 2014;18:352-68.

26 McDonald MD, Sandsmark DK, Palakshappa JA, et al. Long-Term outcomes after extracorporeal life support for acute respiratory failure. J Cardiothorac Vasc Anesth 2019;33:72-9.

27 Rodríguez-Rey R, Alonso-Tapia J. Predicting posttraumatic growth in mothers and fathers of critically ill children: a longitudinal study. $J$ Clin Psychol Med Settings 2019;26:372-81.

28 Rodríguez-Rey R, Palacios A, Alonso-Tapia J, et al. Posttraumatic growth in pediatric intensive care personnel: dependence on resilience and coping strategies. Psychol Trauma 2017;9:407-15.

29 Barreto BB, Luz M, Rios MNdeO, et al. The impact of intensive care unit diaries on patients' and relatives' outcomes: a systematic review and meta-analysis. Crit Care 2019;23:411.

30 Kon AA, Davidson JE, Morrison W, et al. Shared decision making in ICUs: an American College of critical care medicine and American thoracic Society policy statement. Crit Care Med 2016;44:188-201.

31 Engelbrecht A, Jobson L. Exploring trauma associated appraisals in trauma survivors from collectivistic cultures. Springerplus 2016:5:1565.

32 Fields B, Rodakowski J, James AE, et al. Caregiver health literacy predicting healthcare communication and system navigation difficulty. Fam Syst Health 2018;36:482-92.

33 RI VK, Deschepper R, Francke AL, et al. Conflicts between healthcare professionals and families of a multi-ethnic patient population during critical care: an ethnographic learning. Crit Care 2015;19:441.

34 Heyland DK, Davidson J, Skrobik Y, et al. Improving partnerships with family members of ICU patients: study protocol for a randomized controlled trial. Trials 2018;19:3.

35 Høye S, Severinsson E. Multicultural family members' experiences with nurses and the intensive care context: a hermeneutic study. Intensive Crit Care Nurs 2010;26:24-32.

36 Degrie L, Gastmans C, Mahieu L, et al. How do ethnic minority patients experience the intercultural care encounter in hospitals? A systematic review of qualitative research. BMC Med Ethics 2017;18.

37 Wong W-T, Phua J, Joynt GM. Worldwide end-of-life practice for patients in ICUs. Curr Opin Anaesthesiol 2018;31:172-8.

38 Markim A, Cabrera-Fernandez DF, Bajoka RM, et al. Impact of a simulation-based communication workshop on resident prepardness for end-of-life communication in the intensive care unit. Crit Care Res Pract 2015;534879.

39 Awdish RL, Buick D, Kokas M, et al. A communications bundle to improve satisfaction for critically ill patietns and their families: a prospective, cohort pilot study. 2017, Journal pain symptom management 53:3,644-649. 39. Hinkle LJ, Bosslet GT, Torke am: factors associated with family satisfaction with end-of-life care in the ICU: a systematic review. Chest 2015;147:82-93.

40 Wilson ME, Krupa A, Hinds RF, et al. A video to improve patient and surrogate understanding of cardiopulmonary resuscitation choices in the ICU. Crit Care Med 2015;43:621-9.

41 Kichko K, Marschall P, Flessa S. Personalized medicine in the U.S. and Germany: awareness, acceptance, use and preconditions for the wide implementation into the medical standard. J Pers Med 2016;6:15.

42 Frey MK, Ellis A, Shyne S, et al. Bridging the Gap: A Priorities Assessment Tool to Support Shared Decision Making, Maximize Appointment Time, and Increase Patient Satisfaction in Women With Ovarian Cancer. J Oncol Pract 2019;9:JOP1900455.

43 Dowd MD. Shared decision-making tools in pediatric acute care: enhancing parent knowledge and trust. JAMA Network Open 2018;1:e182410.

44 Ernecoff NC, Witteman HO, Chon K, et al. Key stakeholders' perceptions of the acceptability and usefulness of a tablet-based tool to improve communication and shared decision making in ICUs. $J$ Crit Care 2016;33:19-25.

45 van der Schaaf M, Beelen A, Nollet F, et al. Postintensive care syndrome, need for recognition, treatment, research, and expansion of included symptoms. Crit Care Med 2012;40:2742-3. author reply 2743. 